\title{
ON THE PROXIMINALITY OF COMPACT OPERATORS WITH RANGE IN $C(S)$
}

\author{
JAROSLAV MACH
}

\begin{abstract}
It is proved that the space of compact operators on $X$ into $C(S)$ is proximinal in the corresponding space of bounded operators, if $X$ is a Hilbert space, a space $l_{p}, 1<p<+\infty$, or the space $c_{0}$.
\end{abstract}

Let $X$ be a Banach space, $E$ a closed subspace of $X, x \in X$. We denote by $P_{E}(x)$ the set of all elements of best approximation of $x$ in $E$, i.e., $P_{E}(x)=\{e$ $\left.\in E ;\|x-e\|=\inf _{y \in E}\|x-y\|\right\} . E$ is said to be proximinal, if $P_{E}(x) \neq \varnothing$ for every $x \in X$.

Recently, many authors have investigated the problem of best approximation of bounded linear operators $B(X, Y)$ on a Banach space $X$ into a Banach space $Y$ by elements of the corresponding space of compact operators $K(X, Y)$ (see, e.g., [2][5]). Although, in some special cases, a lot of information is known, the following problem still remains open, even for a number of standard Banach spaces: For which Banach spaces $X$ and $Y$ is $K(X, Y)$ proximinal in $B(X, Y)$ ? The purpose of this paper is to give an affirmative answer to this question in the case that $X$ is a Hilbert space, a space $l_{p}, 1<p<+\infty$, or the space $c_{0}$, and $Y$ is $C(S)$, the space of all continuous scalar functions on a compact Hausdorff space $S$.

To obtain one general proof valid for all mentioned cases we make the following definitions.

Definition 1. A Banach space $X$ is said to have the property $\left(\mathrm{P}_{1}\right)$ if there is a continuous strictly increasing function $\Psi: \mathbf{R}^{+} \rightarrow \mathbf{R}^{+}$with $\Psi(0)=0$ and $\Psi(1)=1$ such that the following is true: For every bounded net $\left\{x_{\alpha}\right\}$ in the dual $X^{*}$ of $X w^{*}$-converging to 0 , every $y \in X^{*}$ and each $\varepsilon>0$ there exists an $\alpha_{0}$ such that

$$
\left|\Psi\left(\left\|x_{\alpha}+y\right\|\right)-\Psi\left(\left\|x_{\alpha}\right\|\right)-\Psi(\|y\|)\right|<\varepsilon
$$

for every $\alpha \geqslant \alpha_{0}$.

Definition 2. A Banach space $X$ is said to have the property $\left(\mathrm{P}_{2}\right)$ if for every bounded net $\left\{x_{\alpha}\right\}$ in $X^{*}$ with $w^{*}$ - $\lim x_{\alpha}=0$ and $\lim \left\|x_{\alpha}\right\|=1$, every $y \in X^{*}$ with $\|y\| \leqslant 1$ and every $\varepsilon>0$ there is a cofinal subnet $\left\{x_{\beta}\right\}$ of $\left\{x_{\alpha}\right\}$ such that for every $\beta$

$$
\left\|x_{\beta}-y\right\| \geqslant 1-\varepsilon .
$$

The following is obvious.

Received by the editors August 12, 1976 and, in revised form, December 27, 1976 and September 23, 1977.

AMS (MOS) subject classifications (1970). Primary 41A50, 41A65; Secondary 47B05, 47A30. 
Proposition 1. Every Banach space with the property $\left(\mathrm{P}_{1}\right)$ has the property $\left(\mathbf{P}_{2}\right)$.

Now we give some examples.

Proposition 2. Every Hilbert space $H$ has the property $\left(\mathrm{P}_{1}\right)$.

Proof. Putting $\Psi(t)=t^{2}, t \in \mathbf{R}^{+}$, for any net $\left\{x_{\alpha}\right\} \subset H w^{*}$-converging to 0 and any $y \in H$, the claim follows from the relation

$$
\Psi\left(\left\|x_{\alpha}+y\right\|\right)=\Psi\left(\left\|x_{\alpha}\right\|\right)+\Psi(\|y\|)+\left(x_{\alpha}, y\right)+\left(y, x_{\alpha}\right) .
$$

Proposition 3. Every space $l_{p}, 1<p<+\infty$, has the property $\left(\mathrm{P}_{1}\right)$. The space $c_{0}$ has the property $\left(\mathrm{P}_{1}\right)$.

Proof. For $1 \leqslant q<+\infty$ put $\Psi(t)=t^{q}$. Let $\varepsilon>0, x_{\alpha}=\left\{\xi_{i}^{\alpha}\right\} \in l_{q}$ with $w^{*}-\lim x_{\alpha}=0$, and $y=\left\{\eta_{i}\right\} \in l_{q}$ be given. Put $M=\sup \left\|x_{\alpha}\right\|$. Since $\Psi$ is uniformly continuous in $[0, M+1]$, there is an $n_{0} \in \mathbf{N}$ such that

$$
\left|\sum_{i=n_{0}+1}^{\infty}\right| \xi_{i}^{\alpha}+\left.\eta_{i}\right|^{q}-\sum_{i=n_{0}+1}^{\infty}\left|\xi_{i}^{\alpha}\right|^{q} \mid<\varepsilon / 4
$$

for every $\alpha$, and such that

$$
\sum_{i=n_{0}+1}^{\infty}\left|\eta_{i}\right|^{q}<\varepsilon / 4
$$

Find an $\alpha_{0}$ such that

$$
|| \xi_{i}^{\alpha}+\left.\eta_{i}\right|^{q}-\left|\eta_{i}\right|^{q} \mid<\varepsilon / 4 n_{0}
$$

for $\alpha \geqslant \alpha_{0}, 1 \leqslant i \leqslant n_{0}$, and

$$
\sum_{i=1}^{n_{0}}\left|\xi_{i}^{\alpha}\right|^{q} \leqslant \varepsilon / 4
$$

for $\alpha>\alpha_{0}$. It follows that

$$
\begin{aligned}
\left|\Psi\left(\left\|x_{\alpha}+y\right\|\right)-\Psi\left(\left\|x_{\alpha}\right\|\right)-\Psi(\|y\|)\right| \\
\quad=\left|\sum_{i=1}^{\infty}\right| \xi_{i}^{\alpha}+\left.\eta_{i}\right|^{q}-\sum_{i=1}^{\infty}\left|\xi_{i}^{\alpha}\right|^{q}-\sum_{i=1}^{\infty}\left|\eta_{i}\right|^{q} \mid<\varepsilon .
\end{aligned}
$$

This completes the proof.

Let $X$ be a Banach space. By the representation theorem VI.7.1 of [1], there is a one-to-one correspondence between $B(X, C(S))$ and $w^{*}$-continuous maps $u: S \rightarrow X^{*}$ such that for $L \in B(X, C(X))$

$$
L x(t)=u(t) x, \quad x \in X, t \in S,
$$

and

$$
\|L\|=\sup _{t \in S}\|u(t)\| .
$$

The operator $L$ is compact iff $u$ is continuous with the norm topology in $X^{*}$.

In the next theorem a lower bound for the distance 


$$
\operatorname{dist}(L, K(X, C(S)))=\inf _{T \in K(X, C(S))}\|L-T\|
$$

of an arbitrary $L \in B(X, C(S))$ to $K(X, C(S))$ is given.

Theorem 1. Let $L \in B(X, C(S))$, where $X$ has the property $\left(\mathrm{P}_{2}\right)$. Let $u$ : $S \rightarrow X^{*}$ be the corresponding $w^{*}$-continuous map. Then we have

$$
\operatorname{dist}(L, K(X, C(S))) \geqslant \sup _{t \in S} \limsup _{s \rightarrow t}\|u(t)-u(s)\| .
$$

Proof. For $t \in S$ denote $d(t)=\lim \sup _{s \rightarrow t}\|u(t)-u(s)\|$. For an operator $T \in K(X, C(S))$ let $v: S \rightarrow X^{*}$ be the corresponding map. We have to show that for every $t \in S$

$$
\|L-T\|=\sup _{s \in S}\|u(s)-v(s)\| \geqslant d(t),
$$

or, in other words, that for every $t \in S$ and every $\varepsilon>0$ there is an $s \in S$ such that

$$
\|u(s)-v(s)\| \geqslant d(t)-\varepsilon .
$$

Suppose that this is not true. Then there are a $t_{0} \in S$ and an $\varepsilon_{0}>0$ such that for every $s \in S$ we have

$$
\|u(s)-v(s)\|<d\left(t_{0}\right)-\varepsilon_{0} .
$$

There is a net $\left\{s_{\alpha}\right\} \subset S$ with $\lim s_{\alpha}=t_{0}$ and

$$
\lim \left\|u\left(s_{\alpha}\right)-u\left(t_{0}\right)\right\|=d\left(t_{0}\right) .
$$

Since $v$ is continuous with the norm topology in $X^{*}$, there is an $\alpha_{0}$ such that for every $\alpha \geqslant \alpha_{0}$ we have

$$
\left\|v\left(s_{\alpha}\right)-v\left(t_{0}\right)\right\|<\varepsilon_{0} / 2 .
$$

Consequently, by (2) and (4),

$$
\begin{aligned}
\left\|u\left(s_{\alpha}\right)-v\left(t_{0}\right)\right\| & \leqslant\left\|u\left(s_{\alpha}\right)-v\left(s_{\alpha}\right)\right\|+\left\|v\left(s_{\alpha}\right)-v\left(t_{0}\right)\right\| \\
& <d\left(t_{0}\right)-\varepsilon_{0} / 2
\end{aligned}
$$

for every $\alpha \geqslant \alpha_{0}$. Denoting for $s \in S$

$$
u_{1}(s)=\left(1 / d\left(t_{0}\right)\right) u(s), \quad v_{1}(s)=\left(1 / d\left(t_{0}\right)\right)(v(s)-u(s)),
$$

we obtain $w^{*}-\lim \left(u_{1}\left(s_{\alpha}\right)-u_{1}\left(t_{0}\right)\right)=0$ and, by $(3), \lim \left\|u_{1}\left(s_{\alpha}\right)-u_{1}\left(t_{0}\right)\right\|=1$. Further, by (5), we have for $\alpha \geqslant \alpha_{0}$

$$
\begin{aligned}
\| u_{1}\left(s_{\alpha}\right)- & u_{1}\left(t_{0}\right)-v_{1}\left(t_{0}\right) \| \\
& =\left(1 / d\left(t_{0}\right)\right)\left\|u\left(s_{\alpha}\right)-u\left(t_{0}\right)-v\left(t_{0}\right)+u\left(t_{0}\right)\right\| \\
& =\left(1 / d\left(t_{0}\right)\right)\left\|u\left(s_{\alpha}\right)-v\left(t_{0}\right)\right\|<1-\varepsilon_{0} /\left(2 d\left(t_{0}\right)\right) .
\end{aligned}
$$

Hence $X$ cannot have the property $\left(\mathrm{P}_{2}\right)$ which contradicts the assumption.

Now we formulate our main result.

THeORem 2. If a Banach space $X$ has the property $\left(\mathrm{P}_{1}\right)$, then $K(X, C(S))$ is proximinal in $B(X, C(S))$. 
Proof. Let $L$ be an arbitrary bounded operator in $B(X, C(S)), u: S \rightarrow X^{*}$ the corresponding $w^{*}$-continuous map. Denote $d(t)=\lim \sup _{s \rightarrow t} \| u(t)-$ $u(s) \|, d=\sup _{t \in S} d(t)$. By Theorem 1 it is sufficient to show that there exists a function $v: S \rightarrow X^{*}$ which is continuous with the norm topology in $X^{*}$ such that

$$
\sup _{t \in S}\|v(t)-u(t)\| \leqslant d .
$$

Since $X$ has the property $\left(\mathrm{P}_{1}\right)$, there is a strictly increasing continuous function $\Psi: \mathbf{R}^{+} \rightarrow \mathbf{R}^{+}$with $\Psi(0)=0$ and $\Psi(1)=1$ satisfying (1). Define a set valued mapping $\Phi: S \rightarrow 2^{X^{*}}$ by

$$
\Phi(t)=\left\{x \in X^{*} ;\|x-u(t)\| \leqslant \Psi^{-1}(\Psi(d)-\Psi(d(t)))\right\} .
$$

To accomplish the proof we need the following two lemmas.

LeMma 1. For every $t \in S$ and every $\varepsilon>0$ there is a neighborhood $U$ of $t$ such that for every $s \in U$ we have

$$
\Psi(d(t)) \geqslant \Psi(d(s))+\Psi(\|u(t)-u(s)\|)-\varepsilon .
$$

Proof. Assume that there is a $t \in S$ and an $\varepsilon_{0}>0$ such that every neighborhood of $t$ contains a point $s$ with

$$
\Psi(d(t))<\Psi(d(s))+\Psi(\|u(t)-u(s)\|)-\varepsilon_{0} .
$$

There is a neighborhood $U_{0}$ of $t$ such that for every $s \in U_{0}$ we have

$$
\Psi(\|u(t)-u(s)\|) \leqslant \Psi(d(t))+\varepsilon_{0} / 6 .
$$

Find an $s_{0} \in U_{0}$ for which (7) holds. Further, there is an $s_{1} \in U_{0}$ such that

$$
\Psi\left(\left\|u\left(s_{0}\right)-u\left(s_{1}\right)\right\|\right) \geqslant \Psi\left(d\left(s_{0}\right)\right)-\varepsilon_{0} / 6,
$$

and, since $u$ is $w^{*}$-continuous, such that

$$
\begin{aligned}
\Psi(\| u(t)- & \left.u\left(s_{0}\right)+u\left(s_{0}\right)-u\left(s_{1}\right) \|\right) \\
& \geqslant \Psi\left(\left\|u(t)-u\left(s_{0}\right)\right\|\right)+\Psi\left(\left\|u\left(s_{0}\right)-u\left(s_{1}\right)\right\|\right)-\varepsilon_{0} / 6 .
\end{aligned}
$$

Consequently, by (7)-(10),

$$
\begin{aligned}
\Psi\left(d\left(s_{0}\right)\right)+ & \Psi\left(\left\|u(t)-u\left(s_{0}\right)\right\|\right)-\varepsilon_{0}>\Psi(d(t)) \\
& \geqslant \Psi\left(\left\|u(t)-u\left(s_{1}\right)\right\|\right)-\varepsilon_{0} / 6 \\
& \geqslant \Psi\left(\left\|u(t)-u\left(s_{0}\right)\right\|\right)+\Psi\left(\left\|u\left(s_{0}\right)-u\left(s_{1}\right)\right\|\right)-2 \varepsilon_{0} / 6 \\
& \geqslant \Psi\left(\left\|u(t)-u\left(s_{0}\right)\right\|\right)+\Psi\left(d\left(s_{0}\right)\right)-\varepsilon_{0} / 2 .
\end{aligned}
$$

It follows that $-\varepsilon_{0} / 2>0$. A contradiction.

Lemma 2. For every $t \in S$ and every $\varepsilon>0$ there is a neighborhood $U$ of $t$ such that for every $s \in U$ and every $x \in X^{*}$

$$
\|x-u(t)\| \leqslant \Psi^{-1}(\Psi(d)-\Psi(d(t)))
$$

implies 


$$
\|x-u(s)\| \leqslant \Psi^{-1}(\Psi(d)-\Psi(d(s)))+\varepsilon .
$$

Proof. Given $\varepsilon>0$ there is, since $\Psi^{-1}$ is uniformly continuous in [0, $\Psi(d)+1]$, a $\delta>0$ such that for every $t_{1}, t_{2} \in[0, \Psi(d)+1] t_{1} \leqslant t_{2}+\delta$ implies

$$
\Psi^{-1}\left(t_{1}\right) \leqslant \Psi^{-1}\left(t_{2}\right)+\varepsilon .
$$

By Lemma 1 there is a neighborhood $U_{1}$ of $t$ such that for every $s \in U_{1}$ we have

$$
\Psi(d(t)) \geqslant \Psi(d(s))+\Psi(\|u(t)-u(s)\|)-\delta / 2
$$

Further, since $u$ is $w^{*}$-continuous, there is a neighborhood $U_{2}$ of $t$ such that

$$
\begin{aligned}
& \Psi(\|x-u(t)+u(t)-u(s)\|) \\
& \quad \leqslant \Psi(\|x-u(t)\|)+\Psi(\|u(t)-u(s)\|)+\delta / 2
\end{aligned}
$$

for every $s \in U_{2}$. Then, by (12) and (13), for each $s \in U=U_{1} \cap U_{2}$

$$
\begin{aligned}
\Psi(\|x-u(s)\|) & =\Psi(\|x-u(t)+u(t)-u(s)\|) \\
& \leqslant \Psi(\|x-u(t)\|)+\Psi(\|u(t)-u(s)\|)+\delta / 2 \\
& \leqslant \Psi(d)-\Psi(d(t))+\Psi(\|u(t)-u(s)\|)+\delta / 2 \\
& \leqslant \Psi(d)-\Psi(d(s))+\delta .
\end{aligned}
$$

Consequently, by (11),

$$
\|x-u(s)\| \leqslant \Psi^{-1}(\Psi(d)-\Psi(d(s)))+\varepsilon, \quad s \in U,
$$

which completes the proof of Lemma 2.

Now, we can accomplish the proof of Theorem 2. It follows from Lemma 2 that $\Phi$ is lower semicontinuous (cf. [7, Proposition 2.1]). Hence, by E. Michael's selection Theorem 3.2" of [7], $\Phi$ has a continuous selection $v$ which obviously fulfils (6). This completes the proof of Theorem 2 .

COROLlary 3. If $X$ is a Hilbert space, a space $l_{p}, 1<p<+\infty$, or the space $c_{0}$, then $K(X, C(S))$ is proximinal in $B(X, C(S))$.

I wish to thank to Professor J. D. Ward for many helpful discussions on this and related subjects.

\section{REFERENCES}

1. N. Dunford and J. T. Schwartz, Linear operators. I, Interscience, New York, 1958.

2. R. B. Holmes and B. R. Kripke, Best approximation by compact operators, Indiana Univ. Math. J. 21 (1971), 255-263.

3. R. B. Holmes, B. Scranton, and J. Ward, Best approximation by compact operators. II, Bull. Amer. Math. Soc. 80 (1974), 98-102.

4. Approximation from the space of compact operators and other M-ideals, Duke Math. J. 42 (1975), 259-269. 
5. Uniqueness of commuting compact approximations, Trans. Amer. Math. Soc. 208 (1975), 330-340.

6. G. Köthe, Topologische lineare Räume. I, Springer-Verlag, Berlin and New York, 1966.

7. E. Michael, Contimuous selections. I, Ann. of Math. (2) 63 (1956), 361-382.

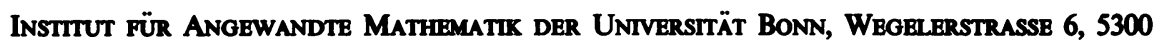
Bonn, Federal Republic of Germany 